\title{
Editorial
}

\section{Kommunikation und Kooperation in Team und Großunternehmen}

Das Thema des vorliegenden Hefts wird in drei Beiträgen und im Kontext mehrerer Polaritäten behandelt: Supervision und Moderation; Team und Organisation; NonProfit- und Profitinstitution; Face-to-Face-Team und Virtuelles Team. Dabei zeigt sich, dass neben begründeten Unterscheidungen auch Übergänge und Wechselwirkungen Beachtung verdienen. Vorsichtige Übertragungen und Generalisierungen sind manchmal möglich: Nicht immer muss das Rad auf jeder Ebene wieder neu erfunden werden.

Reiner Büch (,Imaginative Erkundung der Organisationskultur in einer Psychiatrischen Tagesklinik") definiert Kultur als gemeinsam strukturierte Handlungszeit und Handlungsbereiche auf dem Hintergrund eines Wertekonsens. Das subjektive Handlungspotential gibt Auskunft über die Einschätzung der Fähigkeit zur Erreichung positiv valenter Ziele. Mit Fantasmen meint er Konstrukte der Ich-Realisierung der Außen- und Innenwelt; wir könnten auch sagen, es handelt sich um Aspekte des Selbstkonzept, die Auskunft über die vermutete Eigenwirksamkeit in inneren und äußeren Handlungskontexten geben. Der Mythos schließlich enthält die kulturellen Ordnungen des Handelns, dass in einer Organisation als angemessen gilt. Imagination ist für Büch das Gesamt der vorauseilenden Vorstellungen über mögliche Entwicklungen eines Prozesses. Die Konnotationsanalyse schließlich ist eine mit der Freudschen freien Assoziation verwandte Methode, die die Beziehung zwischen Person und Imagination sichtbar macht. - Die Supervision in Organisationen hat mit allen diesen Konzepten zu tun. Es geht hier nach Büchs Auffassung um die Behebung eines Ordnungsdefizits: Die Divergenz zwischen persönlichem Handlungsmotiv und kollektiver Erwartung oder zwischen unbewusstem Handlungsimpuls und bewusster Intention bedürfen der Sichtbarmachung und Überbrückung. Anhand von zwei Supervisionssitzungen und zahlreichen Einzelinterviews illustriert der Autor: Die SupervisandInnen produzieren nach minimaler Instruktion ein reichhaltiges Material an Fantasien, Gedanken, inneren Bildern über die Institution, über die Zusammenarbeit, über sich selbst und über die Interaktion, die für die weitere Selbsterfahrungs-, Therapie-, und Supervisionsarbeit genutzt werden kann.

Katja Müller (,,Möglichkeiten einer abgestimmten internen Unternehmenskommunikation") untersucht Ist-Stand und Entwicklungsmöglichkeiten der Unternehmenskommunikation. Dabei ist von besonderer Bedeutung, dass Manager in Befragungen die Einschätzung äußern, ihre Arbeit bestehe zu über $80 \%$ aus Kommunikation, und zugleich fast ausnahmslos die schlechte Qualität der betrieblichen Kommunikation beklagen. Für eine eventuelle Besserung dieser Ausgangslage kommt dem mittleren Management eine Schlüsselfunktion zu, weil es die Beschlüsse des Vorstands in Weisungen für die Abteilungen im operativen Geschäft übersetzen muss, oft ohne jede beson- 
dere Qualifikation auf diesem Gebiet. Katja Müllers Untersuchung kann u. a. zeigen, dass eine firmenintern abgestimmte Kommunikation nahezu einhellig gewünscht wird, die bisherigen Versuche auf diesem Gebiet aber überwiegend als unzulänglich eingeschätzt werden.

Dana Unger und Erich Witte (,Virtuelle Teams - geringe Kosten, geringer Nutzen? Zur Leistungsverbesserung von Kleingruppen beim Problemlösen durch elektronische Moderation“) prüfen, ob die Vorteile, die der Zusammenarbeit in Teams in der Fachliteratur überwiegend zugesprochen werden, auch in virtuellen Teams zum Tragen kommen, die im Internet aufgabenbezogen kommunizieren und dabei elektronisch moderiert werden. Sie relativieren zunächst in einer kompakten Übersicht die Gültigkeit der Eingangsprämisse und stellen dann eine eigene experimentelle Untersuchung vor. In den Ergebnissen ergibt sich eine partielle Bestätigung der Vorteilshypothese. Ein methodenund transferkritischer Ausblick bildet den Abschluss der Arbeit.

Der allgemeine Teil dieses Heftes setzt sich wie auch die zwei folgenden Hefte mit dem Thema „Respekt“" auseinander. Dieser Versuch einer Themenreihe wurde von Prof. Dr. Witte und Dr. Quaquebeke gestaltet und erscheint in englischer Sprache. Wir bitten die Leser um Rückmeldung, inwiefern diese Gestaltung auf Zustimmung trifft. 